\title{
Ipilimumab was safe and effective in two patients with metastatic melanoma and end-stage renal disease
}

This article was published in the following Dove Press journal:

Cancer Management and Research

19 January 2015

Number of times this article has been viewed

\section{Ludimila Cavalcante'}

Asim Amin²

Jose Lutzky'

'Department of Hematology and Oncology, Mount Sinai Medical

Center, Miami Beach, FL, USA; 'Levine

Cancer Institute, Charlotte, NC, USA

Correspondence: Jose Lutzky

4300 Alton Road, Miami Beach, FL, USA

Tel +I 3055353300

Fax +I 3055353356

Email jose.lutzky@msmc.com
Abstract: This is a unique report of two cases of patients with end-stage renal disease on hemodialysis, receiving ipilimumab for treatment of metastatic melanoma, as there is a paucity of safety and efficacy data in this patient subgroup.

Keywords: report of cases, hemodialysis, advanced melanoma

\section{Introduction}

Ipilimumab is a fully human IgG-1 antibody that binds to the CTLA-4 molecule a coinhibitory immune checkpoint expressed on activated T cells. Blocking signaling through the CTLA-4 molecule results in unrestrained activation of T cells, which can translate into meaningful clinical response. Ipilimumab is the first agent to have demonstrated a survival advantage in the setting of metastatic melanoma. ${ }^{1,2}$ The US Food and Drug Administration (FDA) approved ipilimumab in 2011 for patients with advanced melanoma, based on the results of a pivotal Phase III trial in previously treated patients. While safety and clinical efficacy data are well-documented for ipilimumab, the Phase III studies excluded patient subgroups with severe renal and hepatic impairment, including patients on chronic hemodialysis. ${ }^{3,4}$

In this report, we describe two patients with end-stage renal disease (ESRD) on hemodialysis who were safely treated with ipilimumab. Both had previously received other treatments for advanced metastatic melanoma. This report will hopefully contribute to the knowledge base on the long-term safety profile of this drug in patients with ESRD, until larger and prospective series become available.

\section{Report of cases Case I}

A 69-year-old Caucasian male initially presented with melanoma in the left posterior auricular area in August 2010. Breslow's thickness was $5.5 \mathrm{~mm}$ with ulceration and one of five sentinel lymph nodes in the left cervical basin were involved. A positron emission tomography-computed tomography (PET/CT) scan revealed no evidence of distant metastatic disease. Left neck dissection performed in October 2010 showed three additional lymph nodes were involved - the final pathologic stage was stage IIIC (T4bN3M0). He had significant comorbidities, including ESRD secondary to glomerulonephritis, for which he had been on hemodialysis since 2009. In the adjuvant setting, he received radiation to the left neck and was not considered fit for systemic intervention due to his comorbidities. 
He remained without evidence of disease recurrence until September 2011, when a PET/CT scan showed several new metabolically active hepatic lesions, as well as small subcentimeter lung nodules consistent with metastatic disease. An ultrasound-guided biopsy of one of the liver lesions confirmed metastatic melanoma. He was treated with temozolomide at a dose of $200 \mathrm{mg} / \mathrm{m}^{2}$ orally for 5 days on a 28-day cycle that he tolerated well. He was treated from September 2011 until repeat imaging in April 2012 showed clear progression of disease. His tumor was found to harbor the BRAF V600E mutation, and he was started on vemurafenib $720 \mathrm{mg}$ orally twice daily, with weekly monitoring due to his ongoing hemodialysis. Restaging PET/CT scan in July 2012 showed a partial response to therapy with a decrease in size of some of the lesions, as well as a reduced standardized uptake value. The response was short-lived, with clear progression in October 2012, followed by discontinuation of vemurafenib (Figure 1).
He was started on immunotherapy with ipilimumab and received four doses of ipilimumab at $3 \mathrm{mg} / \mathrm{kg}$ over 12 weeks. He was clinically noted to have a response in the dermal deposits as early as the second dose of treatment. He developed grade 1 pruritus after his second dose of ipilimumab that was managed with topical over-the-counter hydrocortisone ointment. Overall, his tolerance remained excellent, and he did not require any other intervention. Imaging obtained in January 2013 showed a significant response, which was confirmed at week 16 . His last imaging studies obtained on May 16, 2013 showed ongoing response (Figure 1). The patient continues to do well on hemodialysis with his Eastern Cooperative Oncology Group (ECOG) performance status preserved at 0 .

\section{Case 2}

A 56 year-old Caucasian male with hypertension, diabetes mellitus, and ESRD requiring hemodialysis for 4 years, was

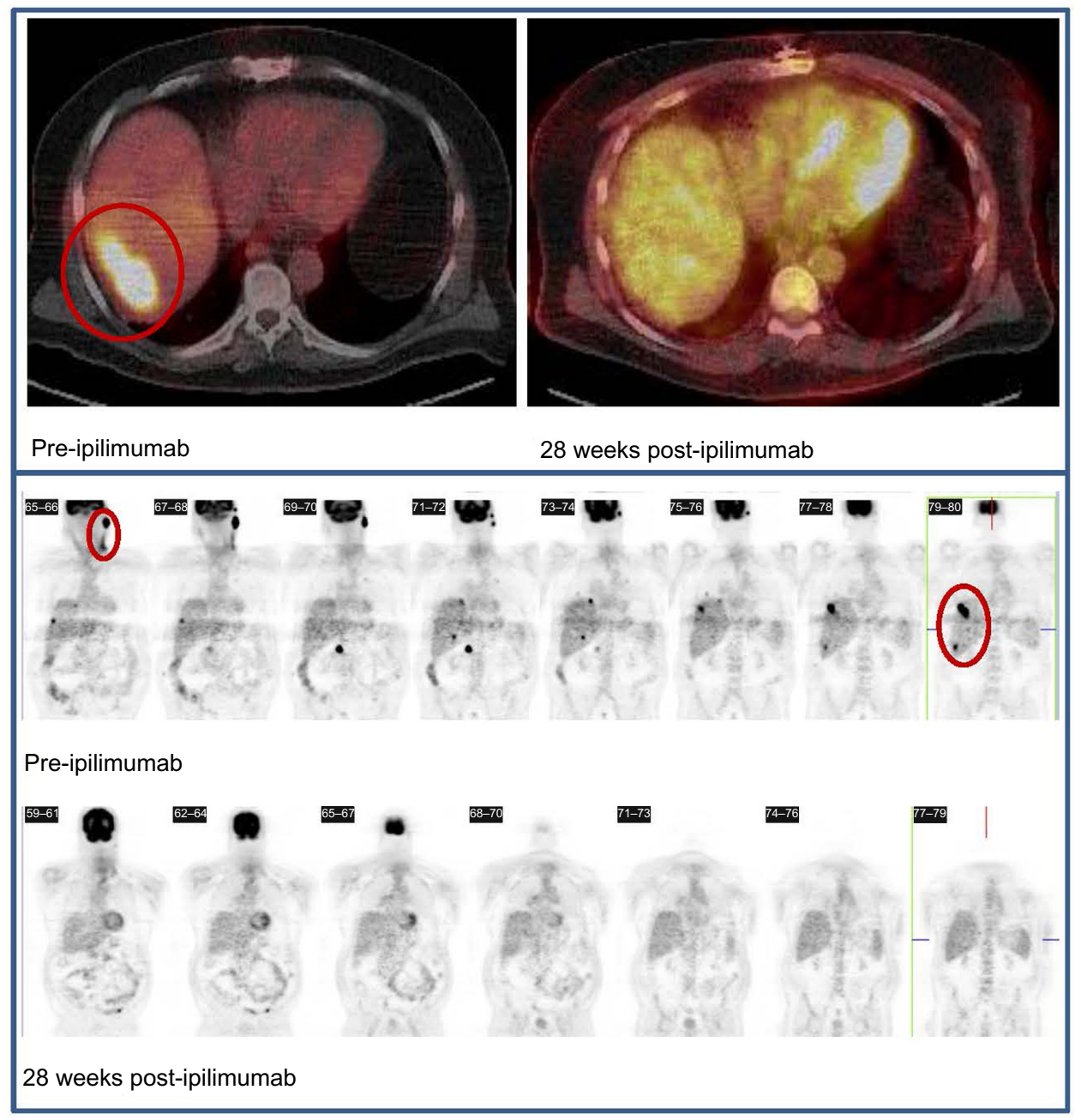

Figure I PET scan done before starting ipilimumab therapy, showing high uptake in the liver region (upper panel) and left cervical chains (lower panel) consistent with metastatic disease in those areas. A repeat scan 28 days after treatment with ipilimumab shows decreased uptake in the same areas, consistent with a positive response to the drug. Abbreviation: PET, positron emission tomography. 
diagnosed in 2010 with a left upper back malignant melanoma, with Breslow's thickness of $2.95 \mathrm{~mm}$, Clark level IV. He underwent wide local excision and sentinel lymph node mapping; the surgical specimen showed extensive residual disease with multiple satellite and in transit metastases involving the dermis and subcutaneous tissues. One sentinel lymph node was positive for metastasis in the left axilla that prompted left axillary dissection and showed 1/15 lymph nodes to be involved. The patient declined adjuvant radiation and interferon at that time. In August 2012, he presented with local recurrence in the form of extensive local subcutaneous involvement that was re-excised. He subsequently developed rapid progression of disease with unresectable multiple satellite and in transit lesions, palpable left cervical lymph nodes, and left axillary lymphadenopathy (Figure 2). His tumor was noted to be BRAF wild-type. His ECOG performance status was 1. Treatment with ipilimumab at $3 \mathrm{mg} / \mathrm{kg}$ was started in November 2012, administered immediately after hemodialysis. After the first dose, the patient developed grade 2 fatigue, grade 2 pruritus, and grade 1 erythematous rash on the left lateral chest wall. His symptoms responded to topical $1 \%$ hydrocortisone cream and oral antihistamines. After the second dose of ipilimumab, marked worsening of skin toxicity was noted, with a grade 3 pemphigoid rash and bullous lesions involving the upper and lower extremities (Figure 2). He was treated with oral systemic steroids, seen by a dermatologist with slow improvement and resolution of the bullous changes, erythema, desquamation, and pruritus. Further doses of ipilimumab were held due to recurrent infections requiring hospitalizations. Restaging computed tomography (CT) scans in January 2013 showed a mixed response, with a new large cytologically negative exudative pericardial effusion, bilateral sub-centimeter pulmonary nodules, and splenomegaly with multiple parenchymal lesions, while the lesions on his left upper back as well as his cervical and axillary lymphadenopathy markedly improved or resolved on physical exam. Subsequent CT scans in March 2013 showed resolution of the pericardial effusion, marked improvement in the pulmonary and splenic lesions, and continuing resolution of the cutaneous and nodal disease. The most recent CT scan from July 2013 revealed essentially no evidence of metastatic disease. Physical exam shows the presence of a few decreasing residual subcutaneous nodules and vitiligo in the upper extremities (Figure 2). His ECOG performance status was 0 and he remains on hemodialysis.

\section{Discussion}

No published data addressing the safety and efficacy of ipilimumab in patients with ESRD on hemodialysis

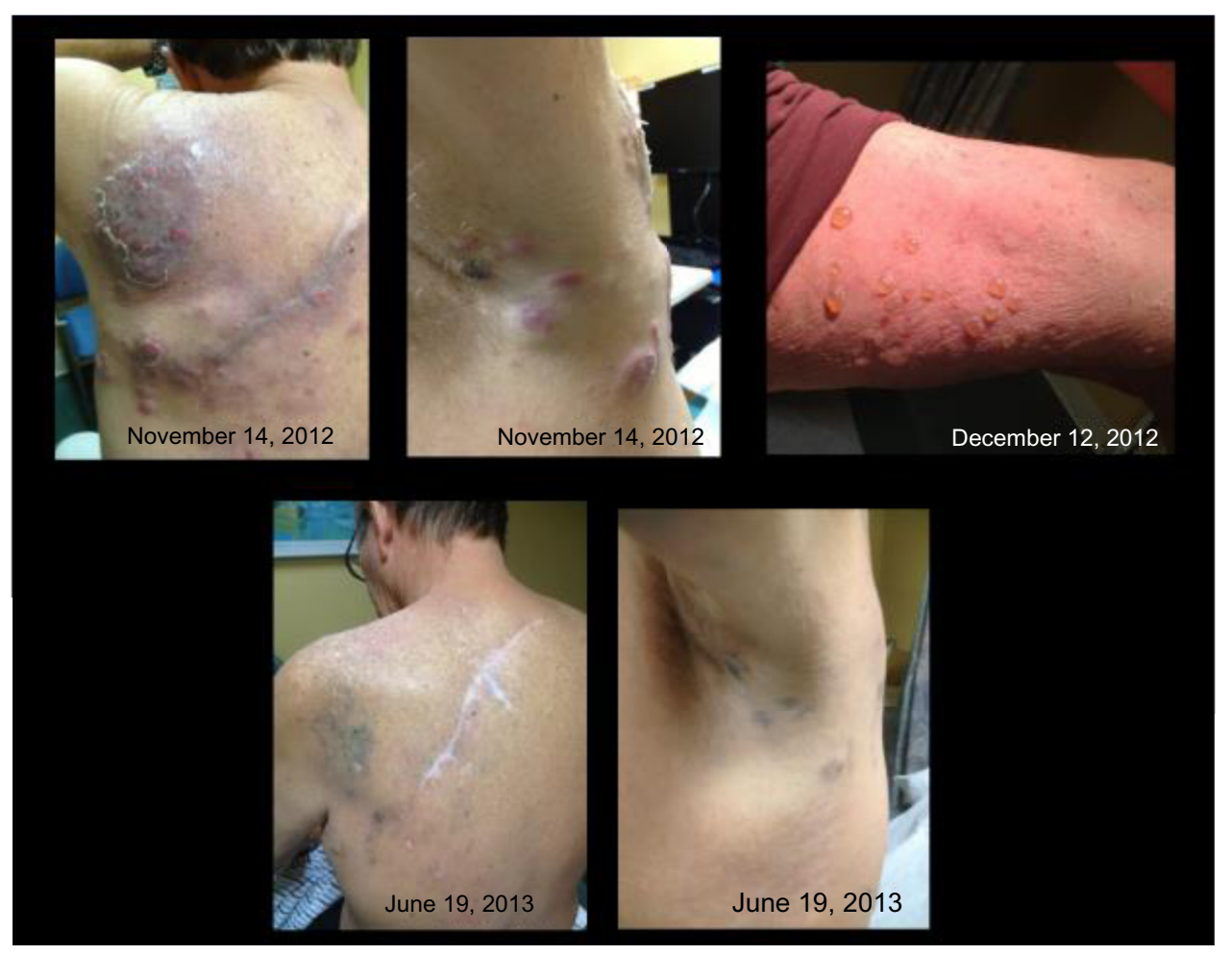

Figure 2 November 14, 20 I2: multiple satellite and in transit lesions on the left lateral back, palpable left cervical lymph nodes and left axillary lymphadenopathy, accompanied by a grade I erythematous rash on the lateral chest wall after the first dose of ipilimumab. December I2, 20I2: grade 3 pemphigoid rash with bullous lesions in the upper extremity. June 19, 2013: currently a few decreasing residual subcutaneous nodules and vitiligo are appreciated in the upper extremities. 
are available. To our knowledge, no studies have been conducted to evaluate the pharmacokinetics of ipilimumab in patients with severe renal impairment. ${ }^{3,5}$ Reports of acute kidney injury and autoimmune nephritis following treatment with ipilimumab have been published in the literature; the incidence is low, affecting less than $2 \%$ of patients receiving treatment. ${ }^{6-8}$ Immune-mediated toxicity is characteristic of ipilimumab-based therapy, and can potentially affect any organ system. ${ }^{9}$ While the concern about worsening renal function may not be relevant in patients with ESRD on hemodialysis, there is paucity of data regarding the safety and efficacy of administering ipilimumab in this subset of patients. Despite the dearth of information regarding its long-term safety profile, administering ipilimumab in this particular population has generally been considered safe by larger centers experienced with its use based on the hepatic metabolism of ipilimumab, lack of renal excretion, and no evidence to suggest tissue accumulation. No dose adjustment is required in the setting of renal impairment, according to the manufacturer's package insert. ${ }^{8}$ Because of its high molecular weight, ipilimumab is not expected to be dialyzed and no dose adjustments were made in our patients.

The two cases described in this report demonstrate that ipilimumab can be administered safely and can elicit clinical benefit in patients with ESRD on hemodialysis. The information from these cases is reassuring, at least until post-marketing prospective studies and pharmacovigilance data become available to validate our observations.

\section{Disclosure}

The authors report no conflicts of interest in this work.

\section{References}

1. Hodi FS, O'Day SJ, McDermott DF, et al. Improved survival with ipilimumab in patients with metastatic melanoma. N Engl J Med. 2010; 363(8):711-723.

2. Robert C, Thomas L, Bondarenko I, et al. Ipilimumab plus dacarbazine for previously untreated metastatic melanoma. N Engl J Med. 2011; 364(26):2517-2526.

3. Hanaizi Z, van Zwieten-Boot B, Calvo G, et al. The European Medicines Agency review of ipilimumab (Yervoy) for the treatment of advanced (unresectable or metastatic) melanoma in adults who have received prior therapy: summary of the scientific assessment of the Committee for Medicinal Products for Human Use. Eur J Cancer. 2012;48(2): 237-242.

4. Garbe C, Peris K, Hauschild A, et al; European Dermatology Forum; European Association of Dermato-Oncology; European Organization of Research and Treatment of Cancer. Diagnosis and treatment of melanoma. European consensus-based interdisciplinary guideline Update 2012. Eur J Cancer. 2012;48(15):2375-2390.

5. Prieto PA, Yang JC, Sherry RM, et al. CTLA-4 blockade with ipilimumab: long-term follow-up of 177 patients with metastatic melanoma. Clin Cancer Res. 2012;18(7):2039-2047.

6. Forde PM, Rock K, Wilson G, et al. Ipilimumab-induced immune-related renal failure - a case report. Anticancer Res. 2012;32(10):4607-4608.

7. Fadel F, El Karoui K, Knebelmann B. Anti-CTLA4 antibody-induced lupus nephritis. $N$ Engl J Med. 2009;361(2):211-212.

8. YERVOY ${ }^{\circledR}$ (ipilimumab) [package insert]. New York, NY: Bristol-Myers Squibb; 2014.

9. Voskens CJ, Goldinger SM, Loquai C, et al. The price of tumor control: an analysis of rare side effects of anti-CTLA-4 therapy in metastatic melanoma from the ipilimumab network. PLoS One. 2013;8(1):e53745.
Cancer Management and Research

\section{Publish your work in this journal}

Cancer Management and Research is an international, peer-reviewed open access journal focusing on cancer research and the optimal use of preventative and integrated treatment interventions to achieve improved outcomes, enhanced survival and quality of life for the cancer patient. The journal welcomes original research, clinical \& epidemiological

\section{Dovepress}

studies, reviews \& evaluations, guidelines, expert opinion \& commentary, case reports \& extended reports. The manuscript management system is completely online and includes a very quick and fair peerreview system, which is all easy to use. Visit http://www.dovepress.com/ testimonials.php to read real quotes from published authors. 